\title{
Vorticity Particle Method for Simulation of 3D Flow
}

\author{
Henryk Kudela and Paweł Regucki \\ Wrocław University of Technology, \\ Wybrzeże Wyspiańskiego 27, \\ 50-370 Wrocław, Poland \\ \{henryk.kudela, pawel.regucki\}@pwr.wroc.pl
}

\begin{abstract}
The vortex-in-cell method for three-dimensional, viscous flow was presented. A viscous splitting algorithm was used. Initially the Euler inviscid equation was solved. Following that, the viscous effect was taken into account by the solution of the diffusion equation. The diffusion equation was then solved by the particle strength exchange (PSE) method. Validation of the method was tested by simulation of the leapfrogging phenomenon for two vortex rings moving along a common axis of symmetry and the reconnection phenomenon of two colliding vortex rings for viscous flow.
\end{abstract}

\section{Introduction}

Interest in computational vortex methods stems from the fact that vorticity plays a fundamental role in all real fluid dynamics phenomena. Vortex particles introduced into the computation permit direct tracking of the vorticity and, additionally, allow for an analysis of the flow phenomena in terms of this vorticity. One can distinguish two different types of vortex methods, the direct method based on the Biot-Savart law where the velocity of each vortex particle is calculated by summing up the contribution of all particles in the domain, and the vortex-in-cell (VIC) method where the velocity is obtained on grid nodes by solving Poisson equations for a vector potential. After that, we differentiate it using the finite difference method, and interpolate the value of the velocity to the position of the vortex particles. Despite the development of fast summation algorithms, VIC methods are still several orders faster than direct methods [1] 4. In the literature one finds that VIC calculations relate mainly to $2 \mathrm{D}$ flow, whereas the extension to 3D flow still requires further investigation.

In this work we validate a $3 \mathrm{D}$ VIC method using examples of vortex ring dynamics: the leap-frogging of two vortex rings, and the reconnection of two colliding vortex rings. The vortex rings are the simplest $3 \mathrm{D}$ vortex structures that can be utilized easily in the laboratory. They are observable in the real turbulent flow. The interaction of two vortex rings gives an interesting and good example of non-linear interaction of the regions with concentrated vorticity and it may serve as a clue to understanding the nature of turbulence. 


\section{Equations of Motion and Description of the Vortex-in-Cell Method}

The equations that describe the evolution of the vorticity field in the three dimensional, incompressible, viscous flow are [2]:

$$
\begin{gathered}
\frac{\partial \boldsymbol{\omega}}{\partial t}+(\boldsymbol{u} \cdot \nabla) \boldsymbol{\omega}=(\boldsymbol{\omega} \cdot \nabla) \boldsymbol{u}+\nu \Delta \boldsymbol{\omega}, \\
\nabla \cdot \boldsymbol{u}=0
\end{gathered}
$$

where $\boldsymbol{\omega}=\left(\omega_{1}, \omega_{2}, \omega_{3}\right)$ is the vorticity vector, $\boldsymbol{u}=\left(u_{1}, u_{2}, u_{3}\right)$ is the velocity and $\nu$ - kinematic viscosity of the fluid. The condition of incompressibility (2) assures the existence of vector potential $\boldsymbol{A}$ [8]:

$$
\boldsymbol{u}=\nabla \times \boldsymbol{A}
$$

where the components of vector potential $\boldsymbol{A}$ are obtained by the solution of the Poisson equations (it is assumed additionally that $\nabla \cdot \boldsymbol{A}=0$ ):

$$
\Delta A_{i}=-\omega_{i}, \quad i=1,2,3
$$

In the vortex-in-cell method, (actually we should speak about the vorticityin-cell method) the continuous field of vorticity is replaced by a discrete distribution of Dirac delta measures [210]:

$$
\boldsymbol{\omega}(\boldsymbol{x})=\sum_{p=1}^{N} \boldsymbol{\alpha}_{p}\left(\boldsymbol{x}_{p}\right) \delta\left(\boldsymbol{x}-\boldsymbol{x}_{p}\right)
$$

where $\boldsymbol{\alpha}_{p}$ means vorticity particle $\boldsymbol{\alpha}_{p}=\left(\alpha_{p 1}, \alpha_{p 2}, \alpha_{p 3}\right)$ at position $\boldsymbol{x}_{p}=$ $\left(x_{p 1}, x_{p 2}, x_{p 3}\right)$. The domain of the flow is covered by the numerical mesh $\left(N_{x} \times N_{y} \times N_{z}\right)$ with equidistant spacing $h$, and the $i$-component of the vector particle $\boldsymbol{\alpha}_{p}$ is defined by the expression:

$$
\alpha_{i}=\int_{V_{p}} \omega_{i}\left(x_{1}, x_{2}, x_{3}\right) d \boldsymbol{x} \approx h^{3} \omega_{i}\left(\boldsymbol{x}_{p}\right), \quad \boldsymbol{x}_{p} \in V_{p}, \quad\left|V_{p}\right|=h^{3} .
$$

In our method, the modelling of the viscous, incompressible flow is based on the viscous splitting algorithm:

- At first the Euler equations of motion for the inviscid flow are solved. From the Helmholtz theorem we know that the vorticity is carried on by the fluid:

$$
\begin{gathered}
\frac{d \boldsymbol{x}_{p}}{d t}=\boldsymbol{u}\left(\boldsymbol{x}_{p}, t\right) \\
\frac{d \boldsymbol{\alpha}_{p}}{d t}=\left[\nabla \boldsymbol{u}\left(\boldsymbol{x}_{p}, t\right)\right] \cdot \boldsymbol{\alpha}_{p} .
\end{gathered}
$$


The right side of (8) may be expressed by virtue of the vector identity as $(\boldsymbol{\omega} \cdot \nabla) \boldsymbol{u}=[\nabla \boldsymbol{u}] \cdot \boldsymbol{\omega}=[\nabla \boldsymbol{u}]^{T} \cdot \boldsymbol{\omega}=0.5\left([\nabla \boldsymbol{u}]+[\nabla \boldsymbol{u}]^{T}\right) \cdot \boldsymbol{\omega}[2]$. We use the term $\left([\nabla \boldsymbol{u}]^{T} \cdot \boldsymbol{\omega}\right)$ because it better preserves the invariants of the motion for the inviscid flow [2]. Velocity and stretching are calculated on the grid nodes by the finite difference method and after that they are interpolated to the position of the particles.

- Next, the strength of the vector particles $\boldsymbol{\alpha}_{p}$, due to the viscosity, is updated:

$$
\frac{d \boldsymbol{\alpha}_{p}}{d t}=\nu \Delta \boldsymbol{\alpha}\left(\boldsymbol{x}_{p}\right)
$$

The Laplacian on the right side of (9) according to the PSE method is replaced by the integral operator, and the equation takes the form:

$$
\frac{d \boldsymbol{\alpha}_{p}}{d t}=\nu \sigma^{-2} \sum_{q=1}^{N}\left(\boldsymbol{\alpha}_{q}-\boldsymbol{\alpha}_{p}\right) \eta\left(\frac{\boldsymbol{x}_{q}-\boldsymbol{x}_{p}}{\sigma}\right)
$$

The kernel $\eta$ must satisfy the moment properties [2]:

$$
\begin{aligned}
& \int x_{i} x_{j} \eta(\boldsymbol{x}) d \boldsymbol{x}=2 \delta_{i j} \quad \text { for } i, j=1,2,3 \\
& \int x_{i}^{i_{1}} x_{j}^{i_{2}} \eta(\boldsymbol{x}) d \boldsymbol{x}=0 \quad \text { if } i_{1}+i_{2}=1 \text { or } 3 \leq i_{1}+i_{2} \leq r+1 \\
& \int|\boldsymbol{x}|^{r+2}|\eta(\boldsymbol{x})| d \boldsymbol{x}<\infty .
\end{aligned}
$$

As a kernel $\eta(\boldsymbol{x})$ we took the function [3]:

$$
\eta(\boldsymbol{x})=\left\{\begin{array}{cc}
\frac{\mathcal{C}}{1+|\boldsymbol{x}|^{2}} & \text { for }|\boldsymbol{x}| \leq 2 \\
0 & \text { for }|\boldsymbol{x}|>2
\end{array}\right.
$$

where $\mathcal{C}=0.269$ was calculated by us in order to satisfy the condition (11). That guarantees that the PSE method is second order. In calculating we took $\sigma=1.5 \mathrm{~h}$. Such a choice of $\sigma$ we made on the basis of a numerical study concerning the dissipation rate of kinetic energy. Equation (10) is solved using the second order Euler scheme.

To solve (41) on the numerical mesh the strength of particles $\boldsymbol{\alpha}_{p}$ has to be redistributed on the mesh nodes $(l, m, n)$ :

$$
\omega_{i}\left(x_{l}, x_{m}, x_{n}\right)=\frac{1}{h^{3}} \sum_{p} \alpha_{i}\left(\boldsymbol{x}_{p}\right) \varphi_{l m n}\left(\boldsymbol{x}_{p}\right), \quad i=1,2,3
$$


where for $\varphi$ we used the $B$-spline of the third order $\varphi_{l m n}(\boldsymbol{x})=\varphi_{l}(x) \varphi_{m}(y) \varphi_{n}(z)$ and the one-dimensional $B$-spline has the form:

$$
\varphi(x)= \begin{cases}\frac{1}{2}|x|^{3}-x^{2}+\frac{2}{3} & \text { for }|x|<1, \\ -\frac{1}{6}|x|^{3}+x^{2}-2|x|+\frac{4}{3} & \text { for } 1 \leq|x| \leq 2, \\ 0 & \text { for }|x|>2 .\end{cases}
$$

The equations (4) are solved by the fast Poisson solver with the periodic boundary conditions.

Summarizing, calculation at one time step $t^{n}=n \Delta t$ goes as follows:

1) redistribution of the particle masses on the grid nodes (15),

2) solution of the Poisson equations (4) and calculations of the velocity on the grid nodes by virtue of (3),

3 ) interpolation of the velocities from the grid nodes to the particle positions by the second order Lagrange interpolation, advancing in time the positions of particles using the fourth order Runge-Kutta method (7) and updating the strength of the particles (8),

4) in the new positions, the strength of the particles is updated due to the viscosity (10).

This completes the one time step.

\section{$3 \quad$ Numerical Results}

As a computational domain for our experiments we chose a cube $10 \times 10 \times 10$ covered by a rectangular grid with equidistant spacing $h=0.1$ in each direction (Fig. 1A). The time step was $\Delta t=0.02$. The single vortex ring was divided into 100 slices (Fig. 1B) and in each slice the vorticity was redistributed on 121 particles (Fig. 1C). Finally, one ring was approximated by the set of 12,100 vector vorticity particles.
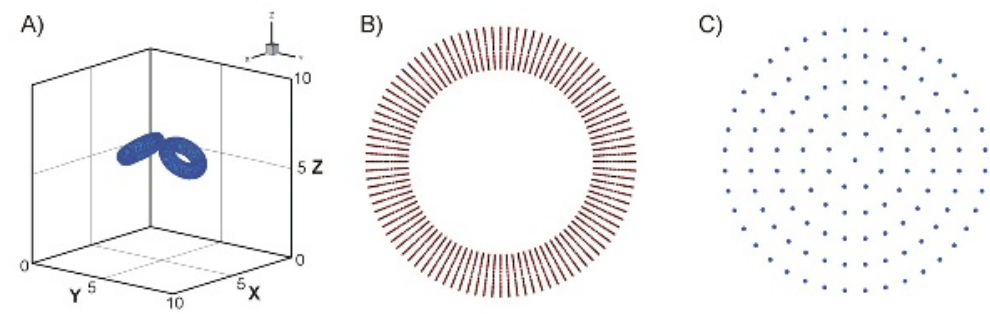

Fig. 1. (A) Computational domain with two vortex rings; $(B)$ division of the vortex ring into 100 slices; $(C)$ initial position of the 121 particles at a single slice 
At first we tried to reproduce the leap-frogging ("vortex game") phenomenon [71114. When two co-axial vortex rings are made to travel in the same direction the velocity field induced by the second ring will cause the first ring to contract and accelerate. At the same time the velocity field induced by the first ring causes an expansion in diameter and a slowing down of the second ring. In effect the first ring is drawn through the center of the second ring and emerges in front of it. When this occurs the roles of the rings are reversed and the process may repeat itself. The numerical results of the simulation of the "vortex game" is presented in Fig. 2. We used two identical vortex rings with a uniform vorticity distribution inside the cores. Their parameters were: radius of the rings $R=1.5$, radius of the cores $\varepsilon=0.15$, circulation $\Gamma=1.0$ and the positions of the ring centers: $(5.0,3.4,5.0),(5.0,4.3,5.0)$.
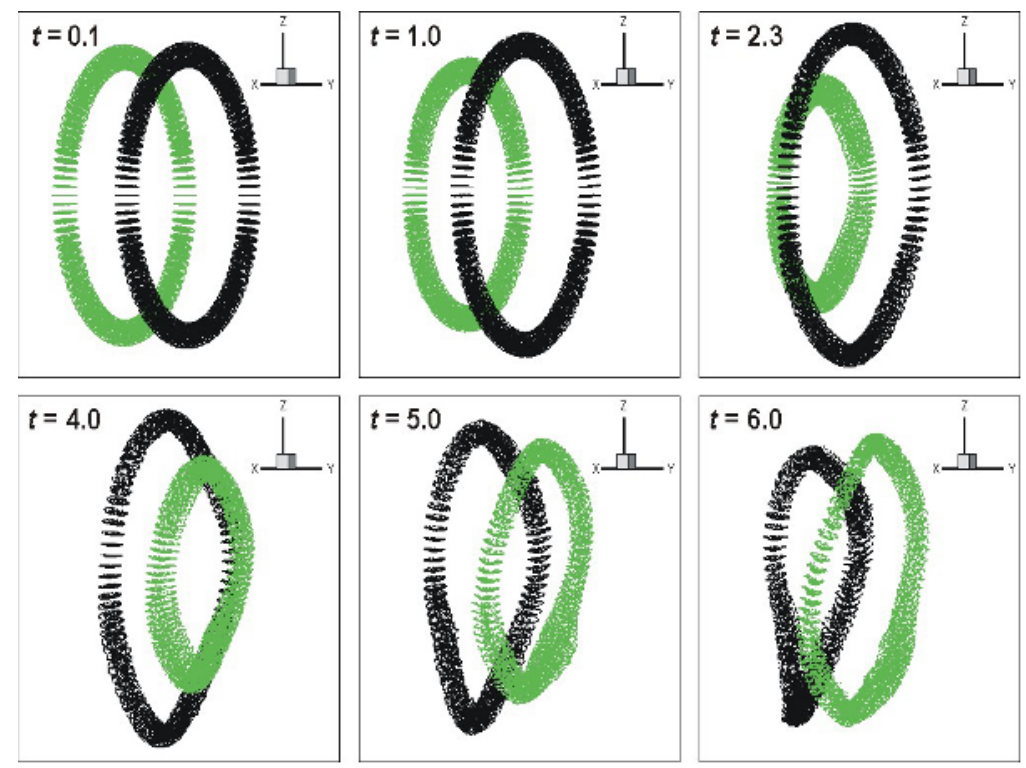

Fig. 2. The sequence of the time position of the vortex particles for the leap-frogging phenomenon in the inviscid flow

In this case we assume that the flow is inviscid. It is known that the "vortex game" is relatively difficult to repeat experimentally [7]. This is due to the fact that it is very sensitive in regard to the initial positions of the rings and their parameters. In Fig. 3 we presented the "vortex game" starting from different initial parameters: radiuses of the rings $R_{1}=1.0, R_{2}=1.5$; radiuses of the cores $\varepsilon_{1}=0.3, \varepsilon_{2}=0.25$; circulations $\Gamma_{1}=1.5, \Gamma_{2}=1.0$; the positions: $(5.0,3.5,5.0),(5.0,4.0,5.0)$. In effect during their evolution the tail structure emerged. It is the most typical situation observed in the experiments [14]. 

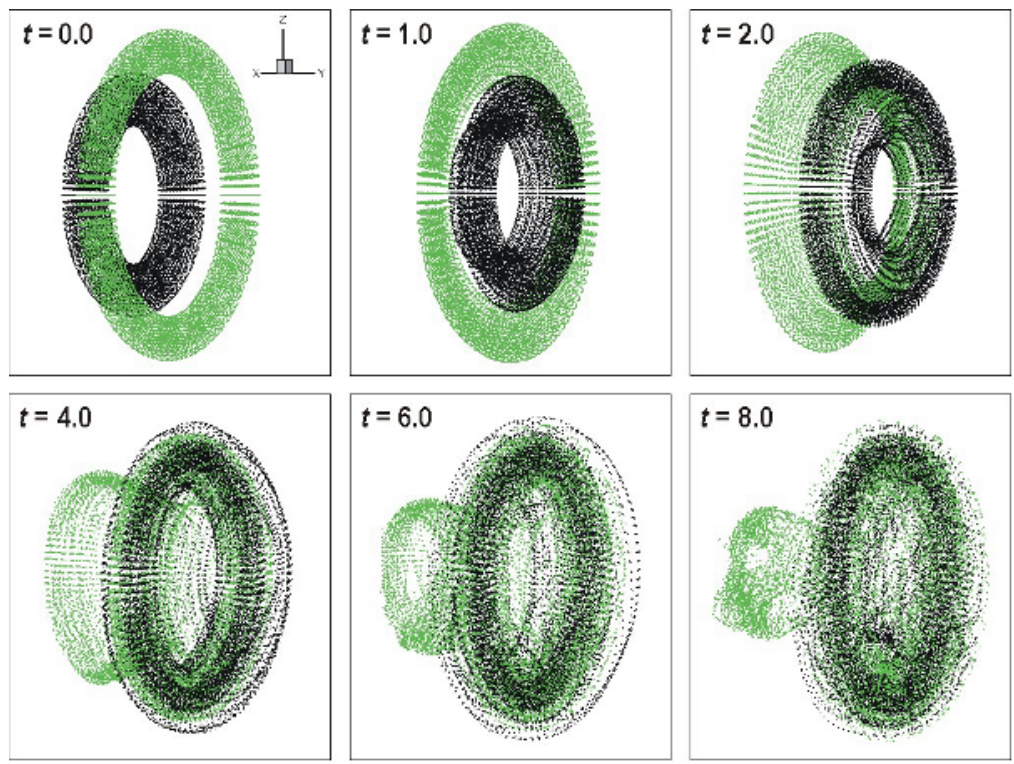

Fig. 3. The sequence of the time position of the vortex particles for the motion of the two rings the "tail structure" in the inviscid flow

Next we studied the reconnection of two vortex rings in viscous flow. It is an intriguing phenomenon (also called cut-and-connect or crosslinking) involving collision of two vortex rings that leads to the changes in connectivity and topology of the vortex rings. It has been extensively studied numerically and experimentally [5613]. We used two identical vortex rings with a uniform vorticity distribution inside the cores. Their parameters were: radius of the rings $R=1.0$, radius of the cores $\varepsilon=0.4$, circulations $|\Gamma|=1.0$ and the positions: $(5.0,3.5,6.0),(5.0,6.5,6.0)$. The initial inclination of the rings to the vertical axis was $54^{\circ}$. Kinematic viscosity was $\nu=0.001$. The sequence of the time position of the vortex particles for the reconnection phenomenon was presented at Fig. 4 In the top diagrams, two rings collided and, in the effect of the first reconnection, formed one elongated ring $(t=15.0)$. Further evolution of this ring led to a second reconnection and in the end there were again two rings connected by the thin filaments (bottom diagrams of Fig. (4). The time evolution of $|\omega|$ iso-surfaces during the reconnection phenomenon was shown at Fig. 5 For better viewing, the bottom diagrams show the vorticity field from a different point of view than the top ones do. In the two top diagrams $(t=4.0, t=10.0)$, a part of vorticity was removed from one ring in order to better show the contact zone. The final diagram clearly shows two vortex rings connected by two thin vortex structures, in literature called "threads" 69. The presented sequence of the vortex rings reconnection process is in good qualitative agreement with the experiment [13]. 

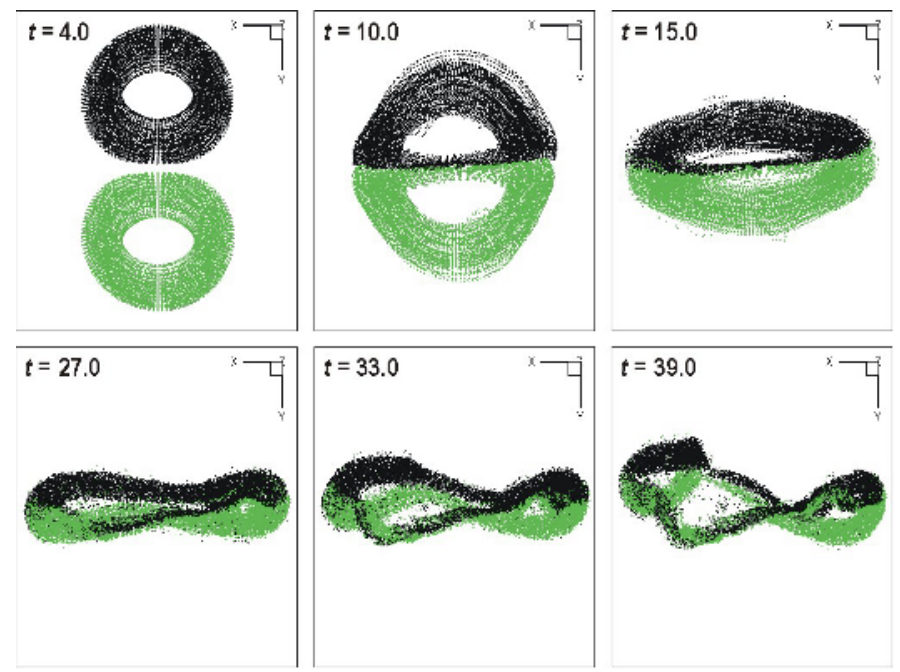

Fig. 4. The sequence of the time position of the vortex particles for the reconnection phenomenon (view from the top)
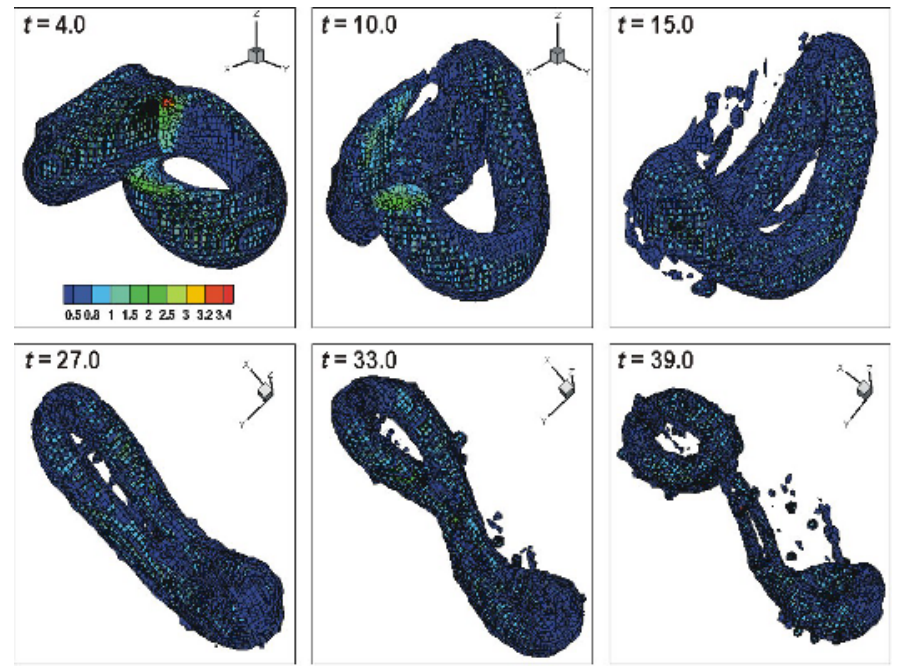

Fig. 5. Time evolution of $|\omega|$ surface during the reconnection phenomenon of two vortex rings. For better viewing, the bottom diagrams show the vorticity field from a different direction than the top ones do 


\section{Closing Remarks}

The presented results indicate that the vorticity particle method is very attractive for studying vortex dynamics phenomena. Compared to the direct vortex method, the vorticity particle method is several orders faster 14. In the near future we intend to include in the algorithm a solid boundary with a no-slip condition, and create a general-purpose program for simulating viscous flow in $3 \mathrm{D}$.

Acknowledgment. This work was supported by The State Committee for Scientific Research under KBN Grant No. 4 T10B 05025.

\section{References}

1. Cottet, G.-H.: 3D Vortex Methods: Achievements and Challenges. In: Vortex Methods, Selected Papers of the First International Conference on Vortex Methods Kobe Japan 1999, ed. K. Kamemoto and M. Tsutahara, World Scientific, (2000) 123-134

2. Cottet, G.-H., Koumoutsakos, P.: Vortex Methods: Theory and Practice. Cambridge University Press, New York (2000)

3. Cottet, G.-H., Michaux, B., Ossia, S., VanderLinden, G.: Comparision of Spectral and Vortex Methods in Three-Dimensional Incompressible Flows. J. Comput. Phys. 175 (2002) 702-712

4. Cottet, G.-H., Poncet, Ph.: Particle Methods for Direct Numerical Simulations of Three-Dimensional Wakes. J. Turbulence. 3(38) (2002) 1-9

5. Kida, S., Takaoka, M.: Vortex Reconnection. Annu. Rev. Fluid Mech. 26 (1994) 169-189

6. Kida, S., Takaoka, M., Hussain, F.: Collision of Two Vortex Rings. J. Fluid Mech. 230 (1991) 583-646

7. Lim, T.T., Nickels, T.B.: Vortex Rings. In: Fluid Vortices, ed. Sh.I. Green, Kluwer Academic Publishers, Dordrecht (1996) 95-153

8. Marshall, J.S.: Inviscid Incompressible Flow. John Wiley \& Sons, Inc., New York (2001)

9. Melander, M.V., Hussain, F.: Reconnection of Two Antiparallel Vortex Tubes: a New Cascade Mechanism. In: Turbulent shear flows 7, Springer-Verlag, Berlin (1991) 9-16

10. Kudela, H., Regucki, P.: The Vortex-in-Cell Method for the Study of ThreeDinemsional Vortex Structures. In: Tubes, Sheets and Singularities in Fluid Dynamics, Vol. 7 of Fluid Mechanics and Its Applications, Kluwer Academic Publisher, Dordrecht (2002) 49-54

11. Oshima, Y., Kambe, T., Asaka, S.: Interaction of Two Vortex Rings Moving along a Common Axis of Symmetry. J. Phys. Soc. Japan. 38(4) (1975) 1159-1166

12. Regucki, P.: Modelling of Three Dimensional Flows by Vortex Methods. Phd Thesis (in polish). Wrocław University of Technology, Wrocław, Poland (2003)

13. Schatzle, P.: An Experimental Study of Fusion of Vortex Rings. Phd Thesis. California Institute of Technology, USA (1987)

14. Yamada, H., Matsui, T.: Mutual Slip-Through of a Pair of Vortex Rings. Phys. Fluids. 22(7) (1979) 1245-1249 